\title{
Role of osteopontin in amplification and perpetuation of rheumatoid synovitis
}

\author{
Guangwu Xu, ${ }^{1}$ Hong Nie, ${ }^{1}$ Ningli Li, ${ }^{1}$ Wenxin Zheng, ${ }^{1}$ Dongqing Zhang, ${ }^{1,2}$ Guozhang Feng, ${ }^{3}$ \\ Liqing $\mathrm{Ni},{ }^{3}$ Rong $\mathrm{Xu},{ }^{3}$ Jian Hong, ${ }^{4}$ and Jingwu Z. Zhang',2,4
}

\begin{abstract}
1Joint Immunology Laboratory of Health Science Center and Shanghai Institute of Immunology, Shanghai Second Medical University and Shanghai Institutes for Biological Sciences, Chinese Academy of Sciences, ${ }^{2} E$-institute of Shanghai Universities, and ${ }^{3}$ Guanghua Rheumatology Hospital, Shanghai, People's Republic of China. ${ }^{4}$ Departments of Immunology and Neurology, Baylor College of Medicine, Houston, Texas, USA.
\end{abstract}

\begin{abstract}
Osteopontin (OPN) is an extracellular matrix protein of pleiotropic properties and has been recently recognized as a potential inflammatory cytokine. In this study, we demonstrate, for the first time to our knowledge, that overexpression of OPN in synovial $\mathrm{T}$ cells is associated with local inflammatory milieu and that OPN acts as an important mediator in amplification and perpetuation of rheumatoid synovitis. The study revealed that mRNA expression of OPN was highly elevated in $\mathrm{CD}^{+}$synovial $\mathrm{T}$ cells derived from patients with $\mathrm{RA}$, which correlated with increased OPN concentrations in synovial fluid (SF). The pattern of OPN overexpression was confined to rheumatoid synovium and correlated with coexpression of selected OPN receptors in synovial T cells, including integrins $\alpha \mathrm{v}$ and $\beta 1$ and CD44. RA-derived SF stimulated the expression of OPN in T cells, which was attributable to IL-10 present in SF and abrogated by anti-IL-10 antibody. Among the more than 300 autoimmune and inflammatory response genes examined, OPN selectively induced the expression of proinflammatory cytokines and chemokines known to promote migration and recruitment of inflammatory cells. Furthermore, it was evident that OPN activated transcription factor NF- $\kappa B$ in mononuclear cells. The study has important implications for understanding the role of OPN in rheumatoid synovitis and other inflammatory conditions.
\end{abstract}

\section{Introduction}

Although the etiology and pathogenesis of RA remains unknown, there is evidence suggesting that $\mathrm{T}$ cell-mediated inflammation plays an important role in rheumatoid synovitis. Several candidate autoantigens have been proposed, such as collagen II, heatshock protein-70, and others (1-4). Their role in the pathogenesis and the disease process remains elusive. However, there is indication that $\mathrm{T}$ lymphocytes, in particular, Th1 cells, and an array of proinflammatory cytokines and chemokines are associated with inflammation and tissue damage in $\mathrm{RA}(5,6)$. Antagonism of TNF- $\alpha$ and its receptors has provided an effective treatment for RA (7-9). However, the molecular mechanisms involved in the activation and perpetuation of inflammatory $\mathrm{T}$ cells in rheumatoid synovium are poorly understood.

Osteopontin (OPN), also known as early T lymphocyte activation-1, has been recently recognized as a potential proinflammatory cytokine associated with inflammatory processes. OPN is an extracellular matrix protein and has pleiotropic functions, including a proinflammatory function $(10,11)$. It is classified as a Th1 cytokine because of its ability to enhance the production of IFN- $\gamma$ and IL-12 in macrophages (10). OPN interacts with a variety of cell surface receptors, including $\alpha v \beta 3, \alpha v \beta 1, \alpha 4 \beta 1, \alpha 8 \beta 1$, and $\alpha 9 \beta 1$ integrins as well as CD44. Binding of OPN to these cell surface receptors induces signaling events that promote cell adhesion and migration (12).

High levels of expression of OPN have been reported in RA and in inflammatory lesions in multiple sclerosis (13-16). The pattern of

Nonstandard abbreviations used: OPN, osteopontin; SF, synovial fluid; SFMC, synovial fluid mononuclear cell; ST, synovial tissue.

Conflict of interest: The authors have declared that no conflict of interest exists.

Citation for this article: J. Clin. Invest. 115:1060-1067 (2005).

doi:10.1172/JCI200523273.
OPN overexpression in T cells present in rheumatoid synovium and the functional role of OPN in rheumatoid synovitis are unknown. It has been speculated that OPN plays an important role in the activation of $\mathrm{T}$ cells and the induction of inflammatory factors. In this study, we hypothesized that OPN expression in synovial $\mathrm{T}$ cells could be induced by cytokine(s) predominantly produced in the inflamed joint. Increased OPN expression may provide a functional mechanism for the perpetuation and amplification of the inflammatory process. We designed experiments first to evaluate the distribution pattern of OPN overexpression in a large panel of well-defined synovial specimens of RA patients and to correlate it with that of cytokines and OPN receptors in $\mathrm{T}$ cells. We then performed analyses to characterize in detail the cytokine(s) that is potentially responsible for the induction of OPN overexpression in rheumatoid synovium and the effect of OPN on the induction of proinflammatory cytokines and chemokines in $\mathrm{T}$ cells. The findings described in this study have provided, for the first time to our knowledge, experimental evidence indicating that OPN plays a central role in the interplay among various cytokines and chemokines, involving the transcription factor NF- $\mathrm{\kappa B}$ pathway, to form an interactive molecular mechanism responsible for amplification and perpetuation of rheumatoid synovitis. The study has important implication for the understanding of the role of OPN in inflammatory process of RA and perhaps in other autoimmune conditions.

\section{Results}

Differential expression of OPN $m R N A$ and its protein levels in rheumatoid synovium and coexpression of receptors for OPN in tissue-derived $T$ cells. PBMCs, synovial fluid (SF) mononuclear cells (SFMCs), and synovial tissue (ST) specimens were obtained from clinically welldefined RA patients and were analyzed and compared with control PBMCs derived from healthy individuals. As shown in Figure 

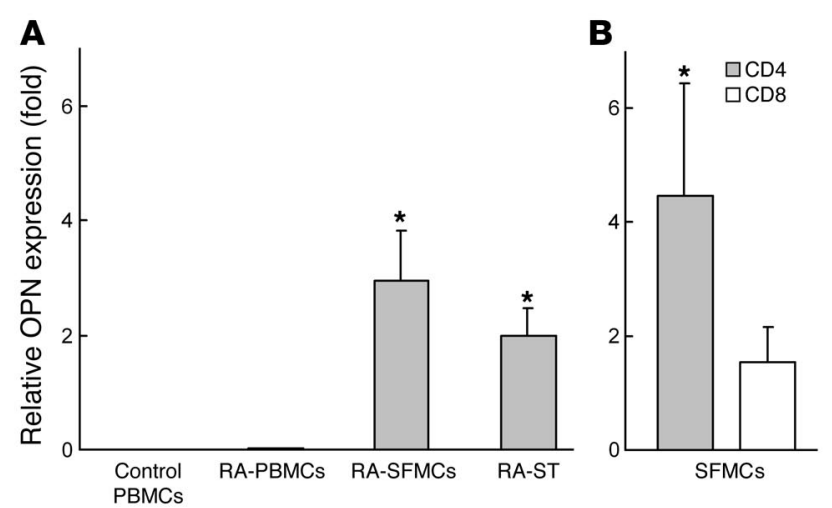

\section{Figure 1}

Expression of OPN mRNA in T cells derived from peripheral blood, SF, and ST. (A) RNA was extracted from T cells isolated from paired SF, ST, and peripheral blood of RA patients $(n=32)$ for real-time PCR analysis. A panel of control T cell preparations was obtained from 31 healthy individuals. OPN expression was normalized to endogenously expressed GAPDH in the same samples. Relative expression was calculated as the difference $\left(\Delta \Delta C_{T}\right)$ between the $\Delta C_{T}$ values of the test sample and of the endogenous control (GAPDH). Relative expression of $O P N$ gene was calculated and expressed as $2^{-\Delta \Delta C T}$ (see Methods). (B) $\mathrm{CD} 4^{+} \mathrm{T}$ cells and $\mathrm{CD} 8^{+} \mathrm{T}$ cells were isolated from the same $\mathrm{T}$ cell preparations of SF specimens by magnetic bead separation and were analyzed for OPN expression by real-time PCR. The purity of the resulting T cell preparations was greater than $97 \%$. The data represent the mean of 6 randomly selected individual T cell preparations. In all cases, asterisks indicate statistically significant differences between the groups $(P<0.05)$.

$1 \mathrm{~A}$, the expression of OPN was significantly elevated $(P<0.05)$, as determined by quantitative PCR analysis, in T cells derived from SF and ST of the same RA patients compared with those in paired PBMCs and control PBMCs. Overexpression of OPN in T cells of SFMCs was found to occur predominantly in purified CD $4^{+} \mathrm{T}$ cell populations (Figure 1B).

As shown in Figure 2, OPN protein was detected at $16.8 \pm 4.3$ $\mu \mathrm{g} / \mathrm{ml}$ in SF derived from RA patients $(n=63)$, a level that was more than 10-fold higher than that in paired RA serum and control serum specimens. The results indicated that the expression of OPN was significantly elevated at both the mRNA and protein levels in synovial $\mathrm{T}$ cells of RA patients. This distribution pattern suggested that the overexpression of OPN was confined to rheumatoid synovium. In parallel analyses by ELISA, concentrations of 5 cytokines, including IL-18, IL-10, IL-12, TNF- $\alpha$, and IFN- $\gamma$ were measured in the same SF and serum specimens of RA patients and control sera. In contrast to IL-18 and IL-12, whose concentrations were significantly elevated in both synovial and serum specimens of RA compared with control sera $(P<0.01)$, IL-10, TNF- $\alpha$, and IFN- $\gamma$ exhibited a distribution pattern similar to that of OPN, which indicated that the increased production of these cytokines was confined to rheumatoid synovium (Figure 3).

OPN interacts with a variety of cell surface receptors, including $\alpha v \beta 3, \alpha v \beta 5, \alpha v \beta 1, \alpha 4 \beta 1, \alpha 8 \beta 1$, and $\alpha 9 \beta 1$ integrins as well as CD44. Binding of OPN to these cell surface receptors mediates the functional properties of OPN on target cells, including $\mathrm{T}$ cells. In this study, coexpression of these receptors in $\mathrm{T}$ cells derived from peripheral blood and synovial T cells was examined by real-time PCR using specific primers (Table 1). As illustrated in Figure 4, some OPN receptors (e.g., $\alpha v, \beta 1$, and CD44) were selectively expressed in T cells derived from RA ST compared with T cells from paired SFMC and PBMC populations $(P<0.01)$. Single-cell suspensions were derived from RA ST specimens and further characterized for $\mathrm{T}$ cell surface expression of positively identified OPN receptors by flow cytometry. Representative expression profiles of integrins $\alpha v$ and $\beta 1$ and CD44 in RA tissue-derived T cells are shown in Figure 5.

Induction of OPN expression by SF and cytokines. Next, we addressed whether the expression of OPN in T cells could be induced by SF that contained various proinflammatory cytokines. PBMC specimens obtained from randomly selected RA patients and healthy volunteers were used for all functional assays described below. PBMC preparations were first exposed in vitro, at the indicated dilutions, to SF of an RA patient. T cells were subsequently isolated from the treated PBMCs and analyzed for mRNA expression of OPN using real-time PCR. As shown in Figure 6A, OPN expression was significantly increased in T cells of RA patients $(n=10)$ in response to RA SF. The observed effect of SF was not specific for $\mathrm{T}$ cells of RA patients, as it was the same for control $\mathrm{T}$ cells derived from 10 healthy individuals. The experiments were reproduced with 4 SF specimens of different RA patients. A dose-response pattern of RA-derived SF on OPN expression is shown in Figure 6B. Similar to what is shown in Figure 1, the increased expression of OPN was found predominantly in the $\mathrm{CD}^{+} \mathrm{T}$ cell subset, as seen in ex vivo analysis of synovial $\mathrm{T}$ cells (data not shown). In contrast, paired sera derived from RA patients did not have the same stimulating effect on OPN expression in T cells (Figure 6B). The stimulating effect of RA-derived SF on the expression of OPN in T cells was significantly inhibited by methotrexate and prednisolone. The specific inhibition rate was $37 \% \pm 7 \%$ for methotrexate $(0.5 \mu \mathrm{g} / \mathrm{ml})$ and $76 \% \pm 13 \%$ for prednisolone $(2 \mu \mathrm{g} / \mathrm{ml})$, respectively.

We further studied whether the inflammatory environment and the cytokine milieu characteristic of rheumatoid synovium could be associated with the overexpression of OPN. To this end, 4 cytokines predominantly present in SF of RA patients (as shown in Figure 3) were evaluated for their ability to stimulate OPN expression in $\mathrm{T}$ cell preparations derived from RA patients. The results indicated that only IL-10, but not the other cytokines tested, activated the expression of OPN in purified T cells (Figure 7A). The

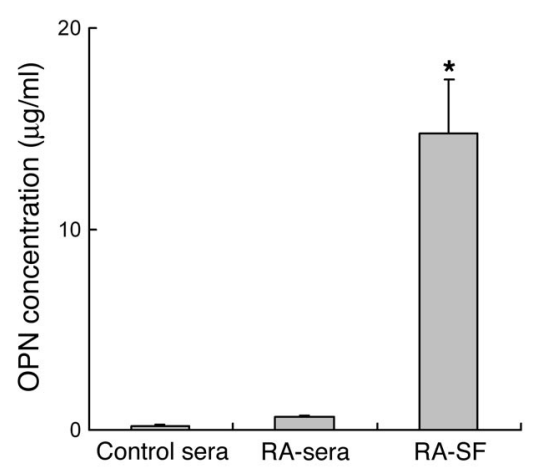

\section{Figure 2}

OPN protein concentrations in SF and serum specimens of RA patients. The OPN protein concentrations were measured by ELISA in 63 paired SF (RA-SF) and serum (RA-sera) samples of RA patients. A panel of 31 serum specimens from healthy individuals was included as a control. Asterisks indicate statistically significant differences between the groups $(P<0.05)$. 

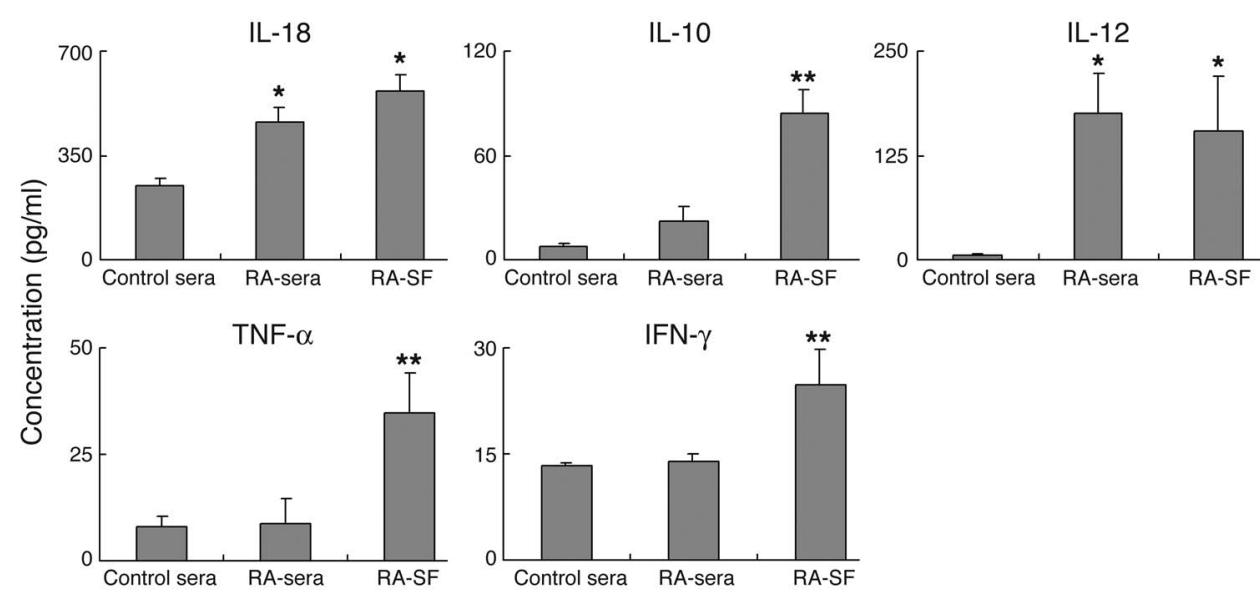

increased expression of OPN after stimulation with IL-10 occurred preferentially in $\mathrm{CD}^{+}$but not $\mathrm{CD}^{+}$subset (data not shown). In addition, the protein concentration of OPN as measured by ELISA was found to rise from baseline to $27.6 \pm 1.1 \mathrm{ng} / \mathrm{ml}$ in $\mathrm{CD}^{+} \mathrm{T}$ cell culture after stimulation with IL-10 as compared with medium control $(<0.05 \mathrm{ng} / \mathrm{ml})$. The dose-response pattern and kinetics of OPN expression in purified T cells in response to IL-10 stimulation are shown in Figure 7B. Further characterization revealed that the observed stimulating effect of SF on T cells could be partially blocked by an antibody against IL-10 but not a control antibody (Figure 7C), which confirmed that IL-10 present in SF was, at least partially, responsible for the induction of OPN expression. Additional experiments showed that the increased OPN expression in $\mathrm{CD}^{+} \mathrm{T}$ cells in response to IL-10 occurred in both $\mathrm{CD}^{2} 5 \mathrm{RA}^{+}$naive and $\mathrm{CD} 45 \mathrm{RO}^{+}$memory $\mathrm{T}$ cell subsets (Figure 7D).

Induction of proinflammatory chemokines and cytokines and activation of transcription factor $N F-\kappa B$ by $O P N$. We were greatly interested in determining whether overexpression of OPN in synovial mononuclear cells could induce the expression of proinflammatory cytokines and chemokines predominantly involved in synovitis. To this end, PBMCs were first exposed to recombinant OPN at a concentration of $1 \mu \mathrm{g} / \mathrm{ml}$ and were analyzed for the expression of 364 genes selected for their involvement in autoimmune and inflammatory responses using a validated cDNA array system. The results indicated that treatment of PBMCs with OPN preferentially induced expression of proinflammatory chemokines and, to a lesser degree, altered expression of proinflammatory cytokine genes (e.g., $I L-1$ and $I L-8$ ) and genes encoding molecules related to the NF- $\mathrm{KB}$ transcription pathway and TNF receptor family (Figure 8A and Table 2). The expression profile of some chemokines and cytokines shown in cDNA array analysis was confirmed by real-time PCR (Figure $8 \mathrm{~B})$. The results were reproduced in additional experiments with different PBMC specimens. Furthermore, we investigated the potential role of transcription factor NF- $\mathrm{KB}$ in the activation of these genes corresponding to proinflammatory chemokines and cytokines, as the NF- $\kappa \mathrm{B}$ pathway is frequently involved in the induction of many proinflammatory cytokine/chemokine genes. As illustrated in Figure 9, OPN had a potent effect on the activation of NF- $\mathrm{KB}$, as evident in the results of a representative electrophoretic mobility shift assay. The observation was confirmed subsequently using a commercially available NF-кB p60 and p65 ELISA kit (data not shown).

\section{Discussion}

The etiology and pathogenesis of RA remains unclear. In addition to the activation of proinflammatory Th1 cells, RA involves many proinflammatory mediators that interact with each other, which constitutes a molecular mechanism that perpetuates and amplifies the inflammatory process in rheumatoid synovium. The importance of inflammatory mediators is exemplified by significant efficacy of TNF- $\alpha$ antagonists in the treatment of RA (17). Characterization of a key inflammatory mediator capable of maintaining such an interactive mechanism in rheumatoid synovitis is even more critical to the understanding of the disease process and the development of novel therapies for RA. This study represents what we believe to be the first systemic investigation to define the expression of OPN in T cells of various tissue sources and to address the functional role and molecular mechanism of OPN in the amplification and perpetuation of inflammation in rheumatoid synovium.

\section{Table 1}

Specific primers used in real-time PCR analysis

\begin{tabular}{|c|c|c|c|}
\hline $\begin{array}{l}\text { Name } \\
\text { (bp) }\end{array}$ & Primer & Sequence $\left(5^{\prime} \rightarrow 3^{\prime}\right)$ & roduct length \\
\hline \multirow[t]{2}{*}{ GAPDH } & FW & GAAGGTGAAGGTCGGAGTC & 187 \\
\hline & RV & GAAGATGGTGATGGGATTTC & \\
\hline \multirow[t]{2}{*}{ OPN } & FW & GCCGACCAAGGAAAACTCACT & 60 \\
\hline & RV & TGCCTAGGAGGCAAAAGCA & \\
\hline \multirow[t]{2}{*}{$\alpha v$} & FW & ACTTCTTGGTGGTCCTGGTAG & 131 \\
\hline & RV & GCAGTCCGAGTTGCTAATTG & \\
\hline \multirow[t]{2}{*}{$\alpha 4$} & FW & CTGGTGGTTGCTATGGAGTG & 190 \\
\hline & RV & AAGAGAGCCAGTCCAGTAAGATG & \\
\hline \multirow[t]{2}{*}{$\alpha 8$} & FW & TTGAAGCTGTCGGCTAGACC & 226 \\
\hline & RV & CACACCACCATCCTGGTTAC & \\
\hline \multirow[t]{2}{*}{$\alpha 9$} & FW & TTAAGTGCCGTGTTCACACC & 193 \\
\hline & RV & GATGTTCTTCCAGCGATGAG & \\
\hline \multirow[t]{2}{*}{$\beta 1$} & FW & CAGGAAGGAATGCCTACTTCTG & 194 \\
\hline & RV & CAACTGCTGTGGTTGGATCTG & \\
\hline \multirow[t]{2}{*}{$\beta 3$} & FW & ACGTGCTGACGCTAACTGAC & 190 \\
\hline & RV & ATGAGTCTTGGCATCAGTGG & \\
\hline \multirow[t]{2}{*}{$\beta 5$} & FW & TCACCTCTCGGTGTGATCTG & 298 \\
\hline & RV & GCTCCGGATATTGTCCAAGTC & \\
\hline \multirow[t]{2}{*}{$C D 44$} & FW & AATGGTCGCTACAGCATCTC & 182 \\
\hline & RV & TGCTGCACAGATGGAGTTG & \\
\hline
\end{tabular}

FW, forward; RV, reverse. 

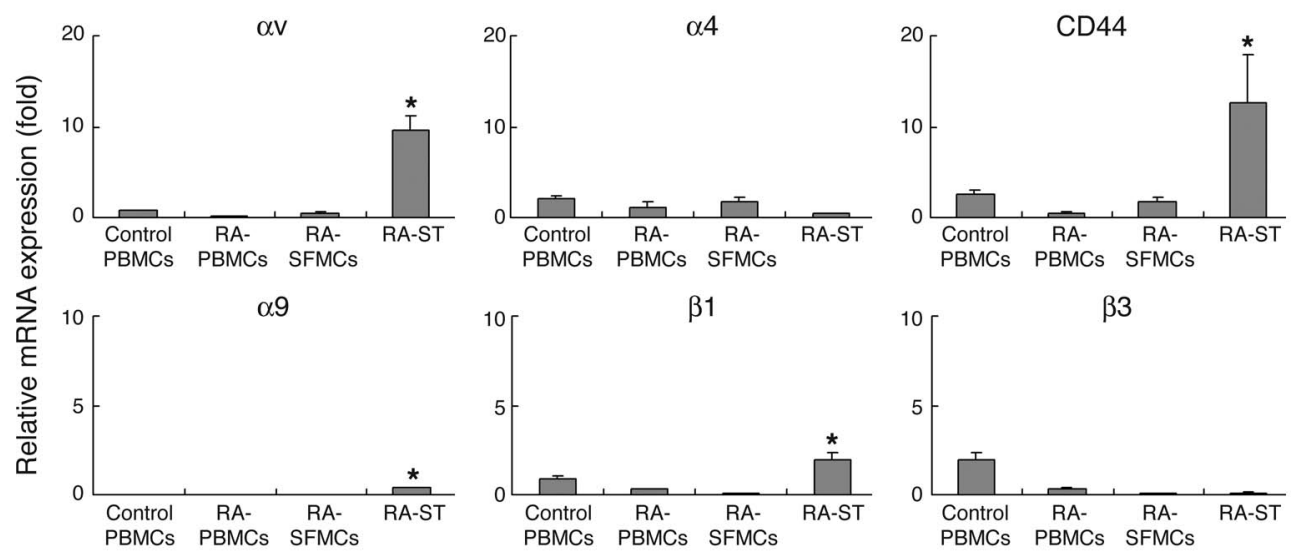

\section{Figure 4}

Coexpression of OPN receptors in T cells isolated from peripheral blood, SF and ST of RA patients. RNA was extracted from isolated T cells of 32 paired ST, SF, and PBMC specimens of RA patients. A panel of 31 control T cell preparations was isolated from healthy individuals. mRNA expression of OPN receptors was normalized to endogenously expressed GAPDH in the same samples. The purity of T cells was greater than $97 \%$ for PBMC and SF specimens and $95 \%$ for ST specimens. Relative expression of OPN receptors was calculated as described in the Figure 1 legend. Asterisks indicate statistically significant differences between the groups $(P<0.01)$.

The findings may have implications regarding the role of OPN not just in RA but in the inflammatory process in general.

Our results show that OPN is significantly overexpressed in synovial $\mathrm{T}$ cells, preferentially in the $\mathrm{CD}^{+}$subset, derived from $\mathrm{RA}$ patients at both the mRNA and protein levels. The OPN protein level can be measured at concentrations of more than $15 \mu \mathrm{g} / \mathrm{ml}$, at least 10 -fold higher than those seen in paired serum specimens of RA patients, and is more than 3 logs higher than synovial levels of many major cytokines predominantly produced in inflamed joint. It is of interest to note that the distribution pattern of OPN overexpression at both the mRNA and protein levels is confined to $\mathrm{T}$ cells of rheumatoid synovium as compared with peripheral blood $\mathrm{T}$ cells in the same RA patients. This distribution pattern is consistent with that of IL-10, TNF- $\alpha$, and IFN- $\gamma$, while the production of IL-18 and IL-12 is not confined to rheumatoid synovium, as they are also detected at similar concentrations in paired sera. Furthermore, we describe, for the first time to our knowledge, differential coexpression of certain, but not all, OPN receptors (e.g., integrins $\alpha v$ and $\beta 1$ and CD44) in the same synovial T cell populations. It is known that OPN interacts with a variety of cell surface receptors, including $\alpha v \beta 3, \alpha v \beta 5, \alpha v \beta 1, \alpha 4 \beta 1, \alpha 8 \beta 1$, and $\alpha 9 \beta 1$ integrins as well as CD44 (14). Binding of OPN to these surface receptors is known to promote cell adhesion and migration and specific signaling events of the host cells. Although a few reports have suggested that some integrins were expressed on RA ST (18-20), we believe that this is the first report comparing the levels of coexpression of OPN receptors in rheumatoid synovial $T$ cells with levels of OPN expression. The observed correlation supports the notion that these receptors are preferentially involved in molecular interactions with OPN in rheumatoid synovium.

Our findings further indicate that OPN overexpression in RA synovium is attributable, at least in part, to IL-10. This conclusion is supported by the observation that IL-10 selectively stimulated OPN expression, which was abrogated by the anti-IL-10 antibody. Although the role of IL-10 in the induction of OPN expression was not originally suspected, as it is an antiinflammatory cytokine, the finding is consistent with significantly elevated IL-10 concentration in SF of RA patients as described here and elsewhere (21,
22) and with the similarity of its distribution pattern to that of OPN. It is likely that OPN and IL-10 interact with each other in a feedback circuit. This is suggested by the observation described here that IL-10 enhances the expression of OPN and other reports showing that OPN inhibits IL-10 production $(10,23)$. These indications collectively suggest the possibility of a negative feedback mechanism that is normally present in maintaining a balance between Th1 immunity (OPN dependent) and Th2 immunity (IL-10 dependent). Potential defects in this homeostatic mechanism may be attributed to the observed cytokine imbalance and inflammation in rheumatoid synovium. On the other hand, IL-10 is a multifaceted cytokine and has a complex role in immune responses, especially in the human system. It has been generally classified as an antiinflammatory cytokine and has a protective effect on experimental arthritis in animal models. However, its role in RA is complex. Treatment with IL-10 yielded obscure results in early RA clinical trials (24). IL-10 has been found to cor-

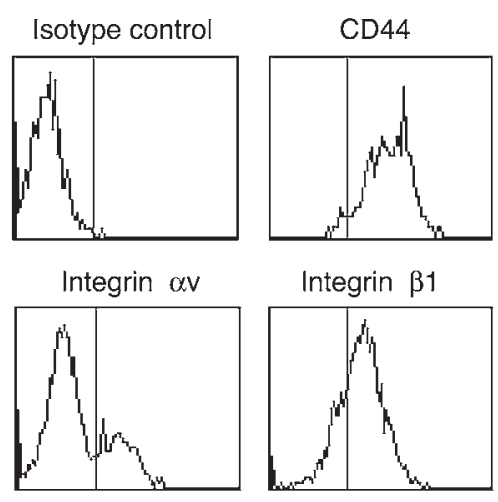

Figure 5

Surface expression of CD44, $\alpha v$ and $\beta 1$ integrins on T cells derived from RA ST. Single-cell suspensions were prepared from RA-ST specimens. The surface expression of CD44 and $\alpha \mathrm{V}$ and $\beta 1$ integrins on gated T cells was analyzed by flow cytometry using specific monoclonal antibodies or an isotype-matched control antibody. The profiles shown were representative of 8 individual sample analyses. 

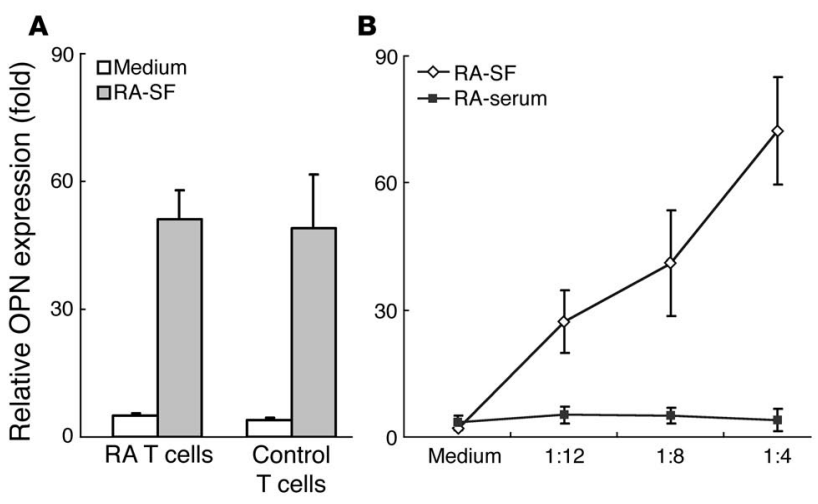

Figure 6

Quantitative real-time PCR analysis of OPN mRNA expression in T cells in response to treatment with RA-SF. (A) Prefiltered SF of an RA patient was added, at a final dilution of 1:5, to cultures of PBMCs derived from 10 randomly selected $R A$ patients and 10 healthy individuals matched for age and sex, respectively. After 48-hour incubation, cells were collected and $\mathrm{CD} 2^{+} \mathrm{T}$ cells were isolated for real-time PCR analysis of OPN expression. The purity of CD2 ${ }^{+} \mathrm{T}$ cells was greater than $97 \%$. The data are representative of 4 separate experiments with SF specimens of different RA patients. (B) The dose-response pattern of OPN expression of the same PBMC specimens of RA patients $(n=10)$ in response to the indicated dilutions of RA SF and paired sera $(n=10)$ under the same experimental conditions as described in $\mathbf{A}$. The results were reproduced with a panel of 10 purified CD4+ $\mathrm{T}$ cell preparations derived from RA patients and healthy individuals. Relative expression of OPN gene was calculated as described in the Figure 1 legend.

relate with autoantibody production and B cell activation in RA, which may contribute to the disease process (25). In this study, our findings indicate that IL-10 does not directly induce expression of chemokines with proinflammatory potential in $\mathrm{T}$ cells but acts indirectly through upregulation of the expression of OPN. However, the results described here do not completely exclude the possibility that other cytokine(s) that were not identified in this study may also contribute to the activation of OPN gene. This is supported by the observation that the stimulating effect of SF on OPN expression was blocked partially by IL-10 antibody.

Furthermore, the findings described here indicate that OPN acts to enhance differential expression of proinflammatory chemokines in PBMCs. With the advantage of using an autoimmune and inflammatory response cDNA array containing 364 selected genes, we demonstrated that OPN preferentially activates some proinflammatory chemokine and cytokine genes (e.g., IL-1, IL-8, CXCL1, CCL2, and so on) among all other autoimmune and inflammatory response

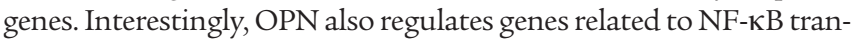
scription pathway and TNF receptor family. We further confirmed that OPN is able to activate the transcription factor NF- $\kappa \mathrm{B}$ pathway, which is known to play a critical role in the production of many inflammatory factors, including cytokines and chemokines (26-28). These demonstrated effects and mechanisms of OPN are essential to the proposed role of OPN in the perpetuation and amplification of inflammatory synovitis. That is, the cytokine milieu produced by inflammatory $\mathrm{T}$ cells and macrophages and enriched for IL-10 in rheumatoid synovium induces overexpression and production of OPN. This further amplifies inflammation through proinflammatory chemokines and cytokines and promotes migration and recruitment of inflammatory cells into inflamed synovi$\mathrm{um}$. This mechanism, mediated by OPN, is likely to play a key role in rheumatoid synovitis. Indeed, Yamamoto and colleagues reported recently that antibody against OPN could inhibit the inflammatory process in arthritic joints in a murine model (29). Further investigations are under way in our laboratory to define the signaling events induced by OPN and potential experimental strategies for the inhibition of OPN-mediated inflammatory process.

\section{Figure 7}

The induction of $O P N$ expression by cytokines and the blocking effect of anti-IL-10 antibody. (A) PBMCs selected from RA patients ( $n=10$, as described in the Figure 6 legend) were cultured in the presence and absence of the indicated cytokines at a final concentration of 25 $\mathrm{ng} / \mathrm{ml}$ for 48 hours. T cells were purified by magnetic bead separation and were subject to real-time PCR analysis of OPN expression. The purity of the isolated T cell preparations was greater than $97 \%$. (B) The same PBMC preparations were cultured in the presence and absence (Medium [control]) of recombinant IL-10 used at the indicated concentrations to determine the dose-response pattern and kinetics of OPN expression in T cells. The resulting cells were harvested at the indicated time points and $T$ cells were purified prior to real-time PCR analysis for OPN expression. (C) In parallel experiments, the same PBMCs (10 untreated samples) were cultured in the presence and absence (Medium [control]) of SF at a dilution of 1:5. A purified mouse anti-human IL-10 antibody or a mouse control antibody of matched isotype (anti-lysozyme antibody), respectively, was added at a final concentration of $5 \mathrm{\mu g} / \mathrm{ml}$. T cells were subsequently isolated and analyzed for OPN expression by real-time PCR. The purity of $T$ cells was greater than $97 \%$. Dash indicates absence of antibody. (D) CD45RA ${ }^{+}$and CD45RO+CD45RA- $T$ cell preparations were purified ( $n=10$; cell purity $>97 \%$ ) using magnetic beads coupled with specific antibodies and analyzed, together with PBMC preparations, for the expression of OPN by real-time PCR. Relative expression of $O P N$ gene was calculated as described in the Figure 1 legend. In all cases, asterisks indicate statistically significant differences between the groups $(P<0.05)$.
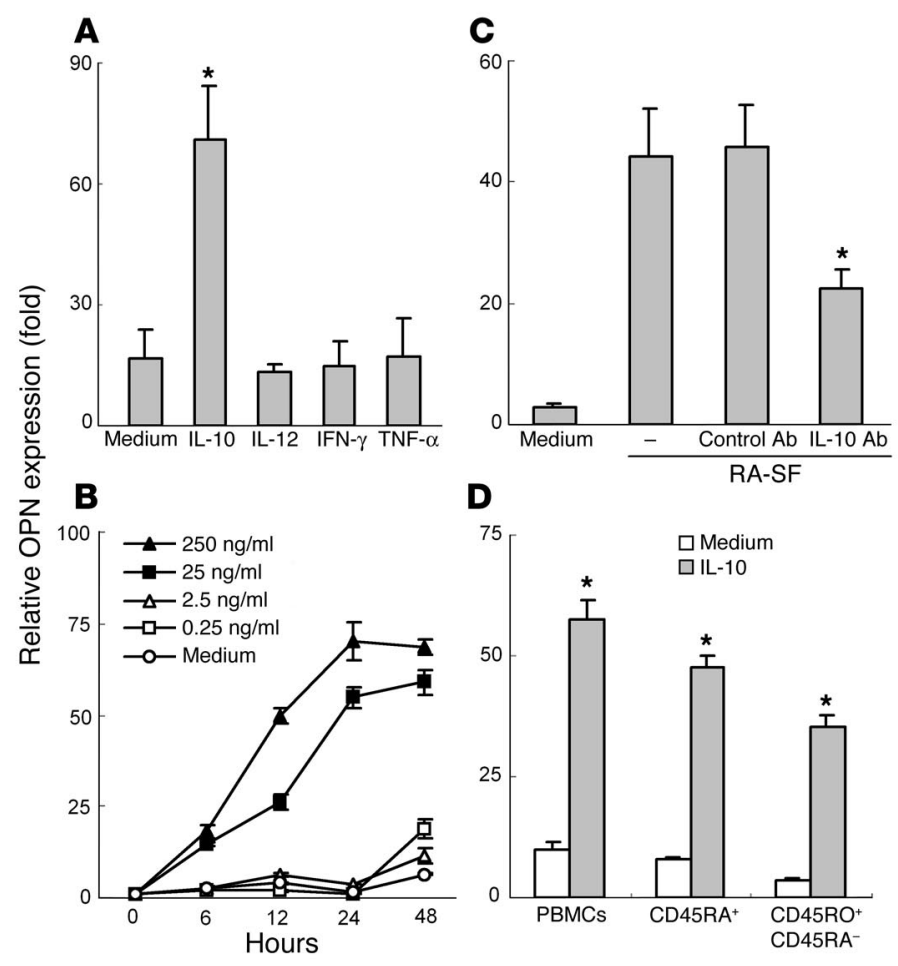
A

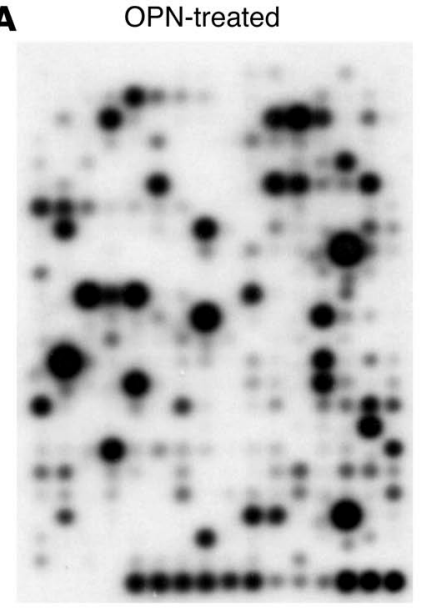

B

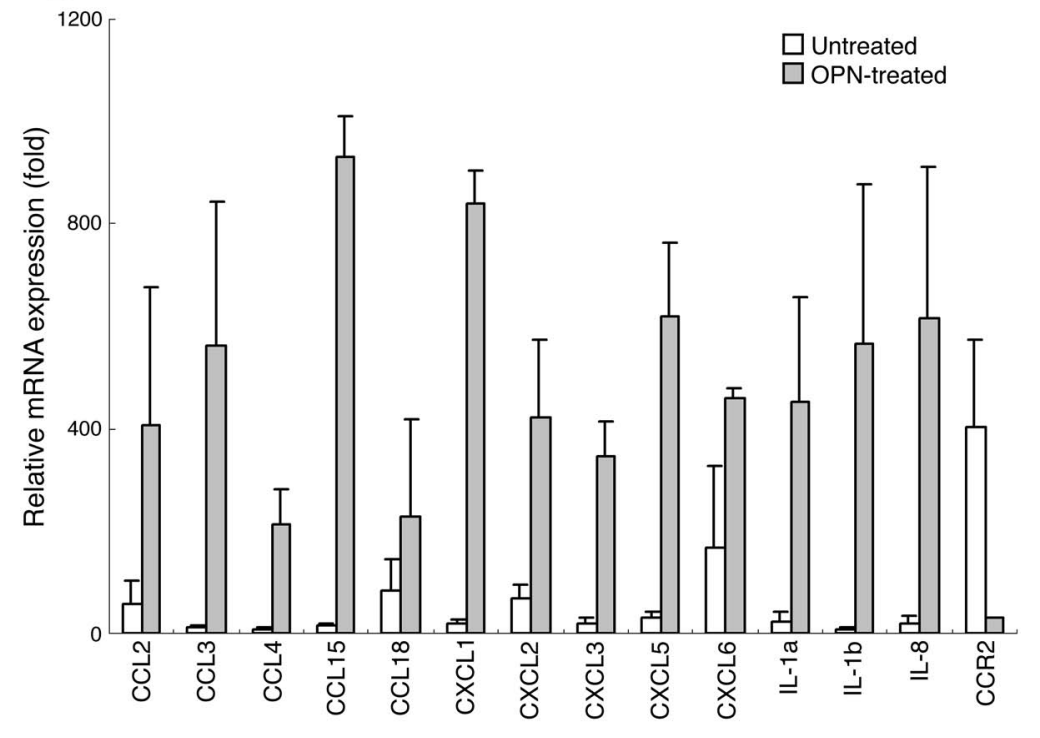

Figure 8

Effect of OPN on the expression of genes corresponding to autoimmune and inflammatory responses. (A) The analyses were performed using an autoimmune and inflammatory response cDNA array system. A representative experiment is shown as dot blots representing the expression profile of selected genes of proinflammatory cytokines and chemokines as indicated at the supplier's website (www.supperarray.com/gene_array_product/ HTML/HS-602.3.html). PBMCs were treated with OPN at $1 \mu \mathrm{g} / \mathrm{ml}$ for 3 hours. The gene expression profile was compared with untreated PBMCs under the same experimental condition. After hybridization with sample cDNA, chemiluminescence was visualized by autoradiography, and digital data were analyzed using Bio-Rad Quantity One software. The results are given as ratio of gene expression of OPN treated to that of untreated controls (see Table 2). (B) PBMC preparations derived from separate RA patients were treated with OPN under the same experimental conditions as described above. The expression of the indicated chemokines and cytokines was analyzed by real-time PCR. Relative expression of the indicated genes was calculated as described in Methods. The differences between OPN treated and untreated control for all chemokines tested were statistically significant $(P<0.01)$.

The tissues were cut into small pieces and immediately homogenized for RNA extraction.

RNA extraction and real-time PCR. Total RNA was isolated from cell pellets using an RNeasy Mini Kit (QIAGEN). Genomic DNA was removed from total RNA prior to cDNA synthesis using the RNase-Free DNase Set for DNase digestion during RNA purification (QIAGEN). RNA was stored at $-80^{\circ} \mathrm{C}$. First-strand cDNA synthesis was performed for each RNA sample using Sensiscript RT Kit (QIAGEN). Random hexamers were used to prime cDNA synthesis.

mRNA expression of OPN, the OPN receptors, and the selected chemokines was determined by real-time PCR using SYBR Green Master Mix (Applied Biosystems). Nucleotide

\section{Methods}

Patients and specimens. A total of 95 patients with RA were included in the study. All patients fulfilled the American College of Rheumatology criteria for RA. This group included 68 females and 27 males with a disease duration of $15 \pm 12$ years. The age of the patients was $54 \pm 19$ years. Complete sets of paired SF, ST, and peripheral blood were obtained from 32 of the 95 patients for paired analyses. Additional sets of synovial and paired serum specimens (no cells) derived from the remaining 63 RA patients were used only for analyses of protein concentrations of OPN and the selected cytokines by ELISA. Control T cells and sera were obtained from a group of 31 healthy individuals matched for sex ratio and mean age with the patient group. At the time of sample collection, patients had not received immunosuppressive or immunomodulatory drugs for at least 2 months. Informed consent was obtained from all study subjects prior to sample collection. The study protocol was approved by the institutional review board of the Health Science Center (Shanghai, China).

Synovial specimens were obtained through synovectomy or arthroscopic procedures that were performed for other medical indications. SF was centrifuged at $350 \mathrm{~g}$ for 3 minutes, and supernatants were collected and immediately stored at $-80^{\circ} \mathrm{C}$ until use. Mononuclear cells were prepared by Ficoll-Hypaque separation (Amersham Biosciences) from SF, and blood specimens of RA patients and were immediately processed for cell culture. sequences of specific primers for OPN and the OPN receptors are listed in Table 1. Thermocycler conditions included an initial holding at $50^{\circ} \mathrm{C}$ for 2 minutes, then $95^{\circ} \mathrm{C}$ for 10 minutes; this was followed by a 2-step PCR program: $95^{\circ} \mathrm{C}$ for 15 seconds and $60^{\circ} \mathrm{C}$ for 60 seconds for 40 cycles. Data were collected and quantitatively analyzed on an ABI PRISM 7900 sequence detection system (Applied Biosystems). The GAPDH gene was used as an endogenous control to normalize for differences in the amount of total RNA in each sample. All values were expressed as fold increase or decrease relative to the expression of GAPDH. The mean value of the replicates for each sample was calculated and expressed as cycle threshold $\left(C_{T}\right.$, cycle number at which each PCR reaction reaches a predetermined fluorescence threshold, set within the linear range of all reactions). The amount of gene expression was then calculated as the difference $\left(\Delta C_{T}\right)$ between the $C_{T}$ value of the sample for the target gene and the mean $C_{T}$ value of that sample for the endogenous control (GAPDH). Relative expression was calculated as the difference $\left(\Delta \Delta C_{T}\right)$ between the $\Delta C_{T}$ values of the test sample and of the control sample. Relative expression of genes of interest was calculated and expressed as $2^{-\Delta \Delta C T}$.

Measurement of SF OPN protein and cytokines by ELISA. Concentrations of OPN protein and the indicated cytokines in serum and SF specimens were measured quantitatively using ELISA kits according to the manufacturer's procedure. OPN ELISA kit was purchased from Assay Designs and was 


\section{Table 2}

Gene expression profile of PBMC after in vitro treatment with OPN

\begin{tabular}{|c|c|c|c|}
\hline Gene groups & $\begin{array}{c}\text { Genes altered } \\
\text { Increased expression }\end{array}$ & Ratio & Position \\
\hline \multirow[t]{10}{*}{ Chemokines } & CCL2 (MCP-1/SCYA2) & 12.9 & 36 \\
\hline & CCL3 (MIP-1 $\alpha / S C Y A 3)$ & 4.5 & 43 \\
\hline & $\operatorname{CCL4}(M I P-1 \beta)$ & 4.0 & 44 \\
\hline & CCL15 (MIP-18/MIP-5) & 2.0 & 31 \\
\hline & CCL18 (PARC) & 3.1 & 34 \\
\hline & CXCL1 (GROa/MGSA) & 5.2 & 86 \\
\hline & 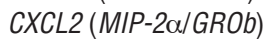 & 2.7 & 91 \\
\hline & CXCL3 (GRO3) & 2.6 & 92 \\
\hline & CXCL5 (ENA-78/SCYA5) & 4.2 & 93 \\
\hline & CXCL6 (GCP-2/SCYB6) & 2.0 & 94 \\
\hline \multirow[t]{3}{*}{ Cytokines } & $I L-1 \alpha$ & 3.4 & 164 \\
\hline & $I L-1 \beta$ & 4.3 & 165 \\
\hline & IL-8 & 2.0 & 184 \\
\hline \multirow{4}{*}{$\begin{array}{l}\text { NF-кB signaling } \\
\text { molecules }\end{array}$} & NFKB1 & 2.0 & 253 \\
\hline & NFKB2 & 2.0 & 254 \\
\hline & NFKBIA & 2.0 & 255 \\
\hline & Decreased expression & & \\
\hline \multirow[t]{4}{*}{ Chemokines/receptors } & CCR2 (MCP-1-R) & 5.0 & 49 \\
\hline & $X C L 1$ (SCYC1) & 2.5 & 358 \\
\hline & XCL2 (SCYC2) & 5.0 & 359 \\
\hline & XCR1 (CCXCR1) & 2.5 & 360 \\
\hline \multirow[t]{2}{*}{ Cytokines } & $I L-11$ & 2.5 & 145 \\
\hline & TGF $\beta 1$ & 3.3 & 310 \\
\hline \multirow{6}{*}{$\begin{array}{l}\text { TNF receptor family } \\
\text { and others }\end{array}$} & TNFRSF8 (CD30) & 3.3 & 337 \\
\hline & TRAF5 & 5.0 & 348 \\
\hline & TRAF6 & 3.3 & 349 \\
\hline & XP05 & 3.3 & 361 \\
\hline & ZFPM2 & 3.3 & 363 \\
\hline & NFAT5 (TONEBP) & 3.3 & 248 \\
\hline
\end{tabular}

designed by the manufacturer to specifically detect the noncleaved form of OPN. The cytokine kits were obtained from Bender MedSystems and BD Biosciences. Microtiter plates precoated with purified mouse antibodies against the indicated cytokines or OPN were washed, and nonspecific binding sites were saturated with $10 \%$ (wt/vol) FBS for 1 hour and subsequently washed. SF or serum, along with the recombinant cytokines as standards, were diluted with PBS and added in duplicate wells. Plates were incubated for 2 hours and subsequently washed with PBS-Tween 20. Matched biotinylated detecting antibody was added and incubated for 2 hours. After washing, avidin-conjugated HRP and 3,3',5,5' -tetramethylbenzidine were used for color development. Optical density was measured and cytokine concentrations were quantitated using Microplate computer software (Bio-Rad Laboratories).

Preparation of single-cell suspension from ST. STs were processed immediately after surgical removal and cut into small pieces in complete RPMI medium

\section{Figure 9}

Activation of transcription factor NF-кB by OPN. The effect of OPN on the activation of NF- $\mathrm{KB}$ in $\mathrm{PBMC}$ preparation derived from an RA patient was analyzed by EMSA. Cells were exposed to recombinant OPN at a concentration of $1 \mu \mathrm{g} / \mathrm{ml}$ for 30 minutes and were subjected to nuclear extraction and EMSA. Lanes 1 and 2, PBMCs treated with OPN; lanes 3 and 4, untreated controls; lane 5, PBMCs treated with TNF- $\alpha$ (40 ng/ $\mathrm{ml}$ ) for 40 minutes. In lanes 2 and 4, 100-fold unlabeled probe of NF-кB was used as a competing agent. The results are representative of 3 separate experiments with different PBMC preparations.
(Invitrogen Corp.) containing 10\% FBS and 1\% penicillin-streptomycin-Lglutamine. Grinding of small pieces of tissue specimens was performed using a micro tissue grinder with a Teflon resin pestle. We obtained singlecell suspension after filtering the sample through nylon mesh of $70 \mu \mathrm{m}$.

Flow cytometric analysis. Cells were resuspended in PBS containing 1\% BSA (Irvine Scientific) and 0.1\% sodium azide (Sigma-Aldrich) and incubated with FITC- or PE-conjugated antibodies or isotype control antibodies at the recommended dilutions for 30 minutes on ice. Antibodies against CD2, $\mathrm{CD} 4$, and CD8 were obtained from BD Biosciences, and antibodies against CD44 and the selected integrins were purchased from Immunotech.

Purification of $T$ cells and isolation of $C D 4^{+}$and $C D 8^{+} T$ cell subsets. Cells were washed by centrifugation in medium and subsequently resuspended in cold PBS containing $2 \%$ FBS at a cell density of 10 million cells per milliliter. We then positively isolated $\mathrm{CD} 2^{+}, \mathrm{CD}^{+}$, and $\mathrm{CD}^{+} \mathrm{T}$ cells using magnetic beads coated with specific antibodies according to the manufacturer's instructions (Dynal Biotech). After separation, the unselected cell fractions were checked for percentage of residual $\mathrm{CD} 2^{+}, \mathrm{CD}^{+}$, and $\mathrm{CD}^{+} \mathrm{T}$ cells by flow cytometry. An aliquot of positively isolated $\mathrm{T}$ cell fraction was detached from beads and analyzed for purity of $\mathrm{CD}^{+}, \mathrm{CD}^{+}$, and $\mathrm{CD}^{+} \mathrm{T}$ cells while the remaining cell preparation was subject to RNA extraction and real-time PCR analysis. In all cases, the residual $\mathrm{T}$ cells were less than $2-3 \%$ and the purity of the resulting $\mathrm{CD}^{+}, \mathrm{CD}^{+}$, and $\mathrm{CD}^{+} \mathrm{T}$ cell preparations was always greater than $97 \%$. In the experiments described here, T cells were isolated following treatment of PBMCs with cytokines (e.g., IL-10) or OPN.

$T$ cell stimulation. PBMCs from RA patients and healthy individuals were cultured in 24-well plates at 1 million cells per well in RPMI 1640 medium containing $20 \%$ FBS in the presence or absence of SF prefiltered through Millex Syringe-Driven Filter Unit (0.22 $\mu \mathrm{m}$; Millipore Corp.) at a dilution of 1:5. Cells were maintained at $37^{\circ} \mathrm{C}$ in a $5 \% \mathrm{CO}_{2}$ atmosphere for 48 hours and were then harvested. T cells were purified for RNA extraction prior to real-time PCR analysis. For blocking experiments, purified monoclonal antibody against human IL-10 (R\&D Systems) or an isotype-matched control antibody was added at a final concentration of $5 \mu \mathrm{g} / \mathrm{ml}$ to the PBMC culture in the presence of SF prior to the procedure mentioned above. The same procedure was employed to examine the inhibitory properties of methotrexate and prednisolone on the expression of OPN in T cells.

To determine the effect of cytokines on the expression of OPN or the effect of OPN on the expression of chemokines, we cultured PBMCs prepared from healthy individuals in 24-well plates at 1 million T cells per well in RPMI 1640 medium containing 20\% FBS in the presence or absence of the indicated cytokines (IL-10 from PeproTech and other cytokines from R\&D Systems) at a final concentration of $25 \mathrm{ng} / \mathrm{ml}$ or purified OPN (Chemicon International) at $1 \mu \mathrm{g} / \mathrm{ml}$. Cells were maintained at $37^{\circ} \mathrm{C}$ and

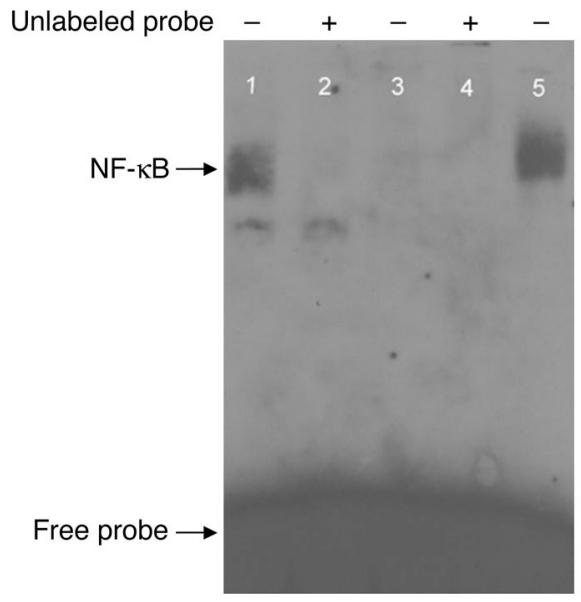


$5 \% \mathrm{CO}_{2}$ for 24 hours and were then harvested for $\mathrm{T}$ cell isolation and RNA extraction prior to real-time PCR analysis.

cDNA array analysis. We examined the expression profile of selected cytokine and chemokine genes using a commercially available cDNA array system containing 364 genes related to autoimmune and inflammatory response and 20 positive and negative control genes (GEArray $S$ Series human autoimmune and inflammatory response gene array; SuperArray Bioscience Corp.) according to the manufacturer's instructions. The gene list is given at the manufacturer's website (www.supperarray.com/gene_array_product/HTML/HS-602.3.html). Briefly, PBMCs were treated with OPN $(1 \mu \mathrm{g} / \mathrm{ml})$ for 3 hours. Total RNA was extracted using TRI Reagent (Molecular Research Center Inc.). Three micrograms of total RNA were reverse transcribed into biotin-16-deoxy-UTPlabeled single-strand cDNA by Moloney murine leukemia virus reverse transcriptase. After prehybridization, membranes were hybridized with biotin-labeled sample cDNA and incubated with alkaline phosphataseconjugated streptavidin. Chemiluminescence was visualized by autoradiography. The results were analyzed using Bio-Rad Quantity One software (Bio-Rad Laboratories). The relative expression of different genes was estimated by comparing signal intensity with that of average intensity of the internal control genes. Data are expressed as ratio of significant change $(\geq 2.0)$ in gene expression (OPN-treated/untreated control). The results are representative of 3 experiments using different PBMC preparations.

$E M S A$. EMSA experiments were done to assess the translocation of NF$\kappa \mathrm{B}$ into nuclei after the treatment of OPN. PBMCs were cultured in the presence or absence of OPN $(1 \mu \mathrm{g} / \mathrm{ml})$ for 40 minutes. TNF- $\alpha$ was used as a positive control. Cell nuclei were prepared using NE-PER Nuclear and Cytoplasmic Extraction Reagents (Pierce Biotechnology Inc.). 5'-AGTT-
GAGGGGACTTTCCCAGGC-3' and its complementary strand were used as the probe. We used a nonradioactive method where the $3^{\prime}$ end of the probe was labeled with biotin for these experiments (Biotin $3^{\prime}$ End DNA Labeling Kit; Pierce Biotechnology Inc.). Biotinylated oligonucleotides were detected with LightShift Chemiluminescent EMSA Kit (Pierce Biotechnology Inc.). Following separation on a $5 \%$ nondenaturing gel, the samples were transferred to a positively charged nylon membrane. The membrane was UV cross-linked, and the biotin end-labeled probe was detected with streptavidin-HRP using a luminol enhancer solution.

Statistics. Differences in the expression of genes between the groups were analyzed by the Mann-Whitney $U$ test. $P$ values of less than 0.05 were considered statistically significant.

\section{Acknowledgments}

The work was supported by grants from the Chinese Academy of Sciences (KSCX2-SW-212), the Chinese Ministry of Science and Technology (863 Project 2002AA216121 and 202CCCD2000), and the Shanghai Commission of Science and Technology (01JC14036, 20014319207, 04DZ14902, and 03XD14015).

Received for publication September 7, 2004, and accepted in revised form January 11, 2005.

Address correspondence to: Jingwu Z. Zhang, Baylor College of Medicine, Mail Station NB302, One Baylor Plaza, Houston, Texas 77030, USA. Phone: (713) 798-3975 or 86-21-63848329; Fax: (713) 798-5665 or 86-21-63852822; E-mail: jzang@bcm.tmc.edu.

Guangwu Xu and Hong Nie contributed equally to this work.
1. Londei, M., et al. 1989. Persistence of collagen type II-specific T-cell clones in the synovial membrane of a patient with rheumatoid arthritis. Proc. Natl. Acad. Sci. U. S. A. 86:636-640.

2. Pope, R.M., Pahlavani, M.A., Lacour, E., Sambol, S., and Desai, B.V. 1989. Antigen specificity of rheumatoid synovial fluid lymphocytes. Arthritis Rheum. 32:1371-1380.

3. Devereux, D., O'Hehir, R.E., McGuire, J., van Schooten, W.C., and Lamb, J.R. 1991. HLA-DR4Dw4restricted $T$ cell recognition of self antigen(s) in the rheumatoid synovial compartment. Int. Immunol. 3:635-640.

4. Res, P.C., et al. 1994. Inflamed joints of patients with rheumatoid arthritis contain $\mathrm{T}$ cells that display in vitro proliferation to antigens present in autologous synovial fluid. Function analysis on the basis of synovial-fluid-reactive T cell clones and lines. Hum. Immunol. 40:291-298.

5. Choy, E.H., and Panayi, G.S. 2001. Cytokine pathways and joint inflammation in rheumatoid arthritis. N. Engl. J. Med. 344:907-916.

6. Szekanecz, Z., Kim, J., and Koch, A.E. 2003. Chemokines and chemokine receptors in rheumatoid arthritis. Semin. Immunol. 15:15-21.

7. Rankin, E.C., et al. 1995. The therapeutic effects of an engineered human anti-tumour necrosis factor alpha antibody (CDP571) in rheumatoid arthritis. Br. J. Rheumatol. 34:334-342.

8. Maini, R., et al. 1999. Infliximab (chimeric antitumour necrosis factor alpha monoclonal antibody) versus placebo in rheumatoid arthritis patients receiving concomitant methotrexate: a randomised phase III trial. ATTRACT Study Group. Lancet. 354:1932-1939.

9. Lipsky, P.E., et al. 2000. Infliximab and metho- trexate in the treatment of rheumatoid arthritis. N. Engl. J. Med. 343:1594-1602.

10. Ashkar, S., et al. 2000. Eta-1 (osteopontin): an early component of type-1 (cell-mediated) immunity. Science. 287:860-864.

11. O'Regan, A.W., Nau, G.J., Chupp, G.L., and Berman, J.S. 2000. Osteopontin (Eta-1) in cell-mediated immunity: teaching an old dog new tricks. Immunol. Today. 21:475-478.

12. Gravallese, E.M. 2003. Osteopontin: a bridge between bone and the immune system. J. Clin. Invest. 112:147-149. doi:10.1172/JCI200319190.

13. Vogt, M.H., Lopatinskaya, L., Smits, M., Polman, C.H., and Nagelkerken, L. 2003. Elevated Osteopontin levels in active relapsing-remitting multiple sclerosis. Ann. Neurol. 53:819-822.

14. Chabas, D., et al. 2001. The influence of the proinflammatory cytokine, osteopontin, on autoimmune demyelinating disease. Science. 294:1731-1735.

15. Ohshima, S., et al. 2002. Enhanced local production of osteopontin in rheumatoid joints. J. Rheumatol. 29:2061-2067.

16. Petrow, P.K., et al. 2000. Expression of osteopontin messenger RNA and protein in rheumatoid arthritis. Arthritis Rheum. 43:1597-1605.

17. Shanahan, J.C., and St. Clair, W. 2002. Tumor necrosis factor-alpha blockade: a novel therapy for rheumatic disease. Clin. Immunol. 103:231-242.

18. Nakayamada, S., et al. 2003. Beta1 integrin-mediated signaling induces intercellular adhesion molecule 1 and Fas on rheumatoid synovial cells and Fas-mediated apoptosis. Arthritis Rheum. 48:1239-1248

19. Johnson, B.A., Haines, G.K., Harlow, L.A., and Koch, A.E. 1993. Adhesion molecule expression in human synovial tissue. Arthritis Rheum. 36:137-146.

20. Walsh, D.A., Wade, M., Mapp, P.I., and Blake, D.R. 1998. Focally regulated endothelial proliferation and cell death in human synovium. Am. J. Pathol. 152:691-702.

21. Cush, J.J., et al. 1995. Elevated interleukin-10 levels in patients with rheumatoid arthritis. Arthritis Rheum. 38:96-104.

22. Furuzawa-Carballeda, J., and Alcocer-Varela, J 1999. Interleukin-8, interleukin-10, intercellular adhesion molecule- 1 and vascular cell adhesion molecule-1 expression levels are higher in synovial tissue from patients with rheumatoid arthritis than in osteoarthritis. Scand. J. Immunol. 50:215-222.

23. Isoda, K., et al. 2003. Osteopontin transgenic mice fed a high-cholesterol diet develop early fattystreak lesions. Circulation. 107:679-681.

24. St. Clair, E.W. 2000. Interleukin-10: therapeutic prospects in rheumatoid arthritis. Curr. Dir. Autoimmun. 2:126-149.

25. Asadullah, K., Sterry, W., and Volk, H.D. 2003. Interleukin-10 therapy--review of a new approach. Pharmacol. Rev. 55:241-269.

26. Muller-Ladner, U., Gay, R.E., and Gay, S. 2002. Role of nuclear factor kappaB in synovial inflammation. Curr. Rheumatol. Rep. 4:201-207.

27. Knight, J.A. 2000. Review: free radicals, antioxidants, and the immune system. Ann. Clin. Lab. Sci. 30:145-158.

28. Richmond, A. 2002. NF-kappa B, chemokine gene transcription and tumour growth. Nat. Rev. Immunol. 2:664-674.

29. Yamamoto, N., et al. 2003. Essential role of the cryptic epitope SLAYGLR within osteopontin in a murine model of rheumatoid arthritis. J. Clin. Invest. 112:181-188. doi:10.1172/JCI200317778. 